\title{
Positive solution of Hilfer fractional differential equations with integral boundary conditions
}

\author{
Mohammed A. Almalahi, Satish K. Panchal and Mohammed S. Abdo
}

Abstract. In this article, we have interested the study of the existence and uniqueness of positive solutions of the first-order nonlinear Hilfer fractional differential equation

$$
D_{0^{+}}^{\alpha, \beta} y(t)=f(t, y(t)), 0<t \leq 1
$$

with the integral boundary condition

$$
I_{0^{+}}^{1-\gamma} y(0)=\lambda \int_{0}^{1} y(s) d s+d,
$$

where $0<\alpha \leq 1,0 \leq \beta \leq 1, \lambda \geq 0, d \in \mathbb{R}^{+}$, and $D_{0^{+}}^{\alpha, \beta}, I_{0^{+}}^{1-\gamma}$ are fractional operators in the Hilfer, Riemann-Liouville concepts, respectively. In this approach, we transform the given fractional differential equation into an equivalent integral equation. Then we establish sufficient conditions and employ the Schauder fixed point theorem and the method of upper and lower solutions to obtain the existence of a positive solution of a given problem. We also use the Banach contraction principle theorem to show the existence of a unique positive solution. The result of existence obtained by structure the upper and lower control functions of the nonlinear term is without any monotonous conditions. Finally, an example is presented to show the effectiveness of our main results.

Mathematics Subject Classification (2010): 34A08, 34B15, 34B18, 34A12, 47H10.

Keywords: Fractional differential equations, positive solution, upper and lower solutions, fixed point theorem, existence and uniqueness.

\section{Introduction}

Fractional differential equations have high significance due to their application in many fields such as applied and engineering sciences, etc. In the recent years, there has been a significant development in ordinary and partial differential equations involving fractional derivatives, see the monographs of Kilbas et al.[8], Miller and Ross [10], Podlubny[12], Hilfer [7] and reference therein. In particular, many interesting results 
of the existence of positive solutions of nonlinear fractional differential equations have been discussed, see $[2,4,5,9,11,13,14]$ and reference therein. The integral boundary conditions have various applications in applied fields such as, underground water flow, blood flow problems, thermo-elasticity, population dynamics, chemical engineering, and so forth. Since only positive solutions are useful for many applications. For example, Abdo et al in [1] discussed the existence and uniqueness of a positive solution for the nonlinear fractional differential equations with integral boundary condition of the form

$$
\begin{array}{lr}
{ }^{c} D_{0^{+}}^{\alpha} y(t)=f(t, y(t)), & t \in[0,1] \\
y(0)=\lambda \int_{0}^{1} y(s) d s+d, & \lambda \geq 0, d \in \mathbb{R}^{+},
\end{array}
$$

where ${ }^{c} D_{0^{+}}^{\alpha}$ is the Caputo fractional derivative of order $\alpha \in(0,1)$, and $f$ satisfied some appropriate assumptions.

Ardjouni and Djoudi in [3], discussed the existence and uniqueness of a positive solution for the nonlinear fractional differential equations

$$
\begin{aligned}
D_{1^{+}}^{\alpha} x(t) & =f(t, x(t)), \quad t \in[1, e] \\
x(1) & =\lambda \int_{1}^{e} x(s) d s+d,
\end{aligned}
$$

where $D_{1+}^{\alpha}$ is the Caputo-Hadamard fractional derivative of order $\alpha \in(0,1), \lambda \geq 0$, $d \in \mathbb{R}^{+}$, and $f$ satisfies some suitable hypotheses. On the other hand, Long et al. [9] investigated some existence of positive solutions of period boundary value problems of fractional differential equations

$$
\left\{\begin{array}{cc}
D_{0^{+}}^{\alpha, \beta} x(t)=\lambda x(t)+f(t, x(t)), & t \in(0, b] \\
\lim _{t \longrightarrow 0^{+}} t^{1-\gamma} x(t)=\lim _{t \longrightarrow b^{-}} t^{1-\gamma} x(0), & \gamma=\alpha+\beta-\alpha \beta
\end{array}\right.
$$

where $\lambda<0, D_{0^{+}}^{\alpha, \beta}$ is the Hilfer fractional derivative of order $\alpha \in(0,1)$ and type $\beta \in[0,1]$ and $f$ satisfied some appropriate conditions.

Motivated by the above works, in this paper, we discuss the existence and uniqueness of positive solution of the following nonlinear Hilfer fractional differential equations with integral boundary condition in a weighted space of continuous functions

$$
\begin{gathered}
D_{0^{+}}^{\alpha, \beta} y(t)=f(t, y(t)), \quad 0<t \leq 1 \\
I_{0^{+}}^{1-\gamma} y(0)=\lambda \int_{0}^{1} y(s) d s+d,
\end{gathered}
$$

where $D_{0^{+}}^{\alpha, \beta}$ is the left-sided Hilfer fractional derivative of order $\alpha \in(0,1)$ of type $\beta \in[0,1], \lambda \geq 0, d \in \mathbb{R}^{+}$and $f:[0,1] \times \mathbb{R}^{+} \longrightarrow \mathbb{R}^{+}$is a continuous, $I_{0^{+}}^{1-\gamma}$ is the Riemann-Liouville fractional integral of order $1-\gamma$, with $\gamma=\alpha+\beta(1-\alpha)$. The Hilfer fractional derivative can be regarded as an interpolator between the RiemannLiouville derivative $(\beta=0)$ and Caputo derivative $(\beta=1)$. Furthermore, there are studies addressed the given problem in cases of $\beta=0,1$, however, to the best of our knowledge, there are no results of the Hilfer problem (1.1)-(1.2), hence, our article aims to fill this gap. 
This article is constructed as follows: In Section 2, we recall some concepts which will be useful throughout this article. Section 3, contains certain sufficient conditions to establish the existence criterions of positive solution by using the Schauder fixed point theorem and the technique of upper and lower solutions. Section 4, demonstrates the uniqueness of the positive solution by using the Banach contraction principle. We are given an example in last section.

\section{Preliminaries}

Let $C_{1-\gamma}[0,1]$ be a weighted space of all continuous function defined on the intervel $(0,1]$, such that

$$
C_{1-\gamma}[0,1]=\left\{y:(0,1] \rightarrow \mathbb{R}^{+} ; t^{1-\gamma} y(t) \in C[0,1]\right\}, 0 \leq \gamma \leq 1
$$

with the norm

$$
\|y\|_{c_{1-\gamma[0,1]}}=\max _{t \in[0,1]}\left|t^{1-\gamma} y(t)\right| .
$$

It is clear that $C_{1-\gamma}\left([0,1], \mathbb{R}^{+}\right)$is Banach space with the above norm. Define the cone $\Omega \subset C_{1-\gamma}[0,1]$ by

$$
\Omega=\left\{y(t) \in C_{1-\gamma}[0,1]: y(t) \geq 0, t \in(0,1]\right\} .
$$

Definition 2.1. [8] The left-sided Riemann-Liouville fractional integral of order $\alpha>0$ with the lower limit zero for a function $y: \mathbb{R}^{+} \longrightarrow \mathbb{R}$ is defined by

$$
\left(I_{0^{+}}^{\alpha} y\right)(t)=\frac{1}{\Gamma(\alpha)} \int_{0}^{t}(t-s)^{\alpha-1} y(s) d s,
$$

provided the right-hand side is pointwise on $\mathbb{R}^{+}$, where $\Gamma$ is the gamma function.

Definition 2.2. [8] The left-sided Riemann-Liouville fractional derivative of order $0<$ $\alpha<1$ with the lower limit zero of a function $y: \mathbb{R}^{+} \longrightarrow \mathbb{R}$ is defined by

$$
D_{0^{+}}^{\alpha} y(t)=\frac{1}{\Gamma(1-\alpha)} \frac{d}{d t} \int_{0}^{t}(t-s)^{\alpha-1} y(s) d s .
$$

provided the right-hand side is pointwise on $\mathbb{R}^{+}$.

Definition 2.3. [8] The left-sided Caputo fractional derivative of order $0<\alpha<1$ with the lower limit zero of a function $y: \mathbb{R}^{+} \longrightarrow \mathbb{R}$ is given by

$$
{ }^{c} D_{0^{+}}^{\alpha} y(t)=\frac{1}{\Gamma(1-\alpha)} \int_{0}^{t}(t-s)^{\alpha-1} y^{\prime}(s) d s .
$$

provided the right-hand side is pointwise on $\mathbb{R}^{+}$.

Definition 2.4. [6] The left-sided Hilfer fractional derivative of order $0<\alpha<1$ and type $0 \leq \beta \leq 1$ with the lower limit zero of a function $y: \mathbb{R}^{+} \longrightarrow \mathbb{R}$ is given by

$$
D_{0^{+}}^{\alpha, \beta} y(t)=I_{0^{+}}^{\beta(1-\alpha)} D I_{0^{+}}^{(1-\beta)(1-\alpha)} y(t),
$$

where $D=\frac{d}{d t}$. One has,

$$
D_{0^{+}}^{\alpha, \beta} y(t)=I_{0^{+}}^{\beta(1-\alpha)} D_{0^{+}}^{\gamma} y(t)
$$


712 Mohammed A. Almalahi, Satish K. Panchal and Mohammed S. Abdo

where

$$
D_{0^{+}}^{\gamma} y(t)=D I_{0^{+}}^{1-\gamma} y(t), \gamma=\alpha+\beta(1-\alpha) .
$$

In the forthcoming analysis, we need the following spaces:

$$
C_{1-\gamma}^{\alpha, \beta}[0,1]=\left\{y \in C_{1-\gamma}[0,1]: D_{0^{+}}^{\alpha, \beta} y \in C_{1-\gamma}[0,1]\right\}
$$

and

$$
C_{1-\gamma}^{\gamma}[0,1]=\left\{y \in C_{1-\gamma}[0,1]: D_{0^{+}}^{\gamma} y \in C_{1-\gamma}[0,1]\right\} .
$$

Since $D_{0^{+}}^{\alpha, \beta} y=I_{0^{+}}^{\beta(1-\alpha)} D_{0^{+}}^{\gamma} y$, it is obvious that $C_{1-\gamma}^{\gamma}[0,1] \subset C_{1-\gamma}^{\alpha, \beta}[0,1]$.

Lemma 2.5. [2] Let $\alpha>0, \beta>0$ and $\gamma=\alpha+\beta-\alpha \beta$. If $y \in C_{1-\gamma}^{\gamma}[0,1]$, then

$$
I_{0^{+}}^{\gamma} D_{0^{+}}^{\gamma} y=I_{0^{+}}^{\alpha} D_{0^{+}}^{\alpha, \beta} y
$$

and

$$
D_{0^{+}}^{\gamma} I_{0^{+}}^{\alpha} y=D_{0^{+}}^{\beta(1-\alpha)} y
$$

Theorem 2.6. [6] Let $y \in C_{\gamma}[0,1], 0<\alpha<1$, and $0 \leq \gamma<1$. Then

$$
D_{0^{+}}^{\alpha} I_{0^{+}}^{\alpha} y(t)=y(t), \forall t \in(0,1] \text {. }
$$

Moreover, if $y \in C_{\gamma}[0,1]$ and $I_{0^{+}}^{1-\beta(1-\alpha)} y \in C_{\gamma}^{1}[0,1]$, then

$$
D_{0^{+}}^{\alpha, \beta} I_{0^{+}}^{\alpha} y(t)=y(t), \text { for a.e. } t \in(0,1] \text {. }
$$

Theorem 2.7. [6] Let $\alpha, \beta \geq 0$ and $y \in C_{\gamma}^{1}[0,1], 0<\alpha<1$, and $0 \leq \gamma<1$. Then

$$
I_{0^{+}}^{\alpha} I_{0^{+}}^{\beta} y(t)=I_{0^{+}}^{\alpha+\beta} y(t)
$$

Lemma 2.8. [8] Let $\alpha \geq 0$, and $\sigma>0$. Then

$$
I_{0^{+}}^{\alpha} t^{\sigma-1}=\frac{\Gamma(\sigma)}{\Gamma(\alpha+\sigma)} t^{\alpha+\sigma-1}, t>0
$$

and

$$
D_{0^{+}}^{\alpha} t^{\alpha-1}=0, \quad 0<\alpha<1
$$

Lemma 2.9. [6] Let $0<\alpha<1,0 \leq \gamma \leq 1$, if $y \in C_{\gamma}[0,1]$ and $I_{0^{+}}^{1-\alpha} y \in C_{\gamma}^{1}[0,1]$, we have

$$
I_{0^{+}}^{\alpha} D_{0^{+}}^{\alpha} y(t)=y(t)-\frac{I_{0^{+}}^{1-\alpha} y(0)}{\Gamma(\gamma)} t^{\alpha-1}, \quad \text { for all } t \in(0,1] \text {. }
$$

Lemma 2.10. [6] Let $y \in C_{\gamma}[0,1]$. If $0 \leq \gamma<\alpha<1$, then

$$
\lim _{t \longrightarrow 0^{+}} I_{0^{+}}^{\alpha} y(t)=I_{0^{+}}^{\alpha} y(0)=0 \text {. }
$$




\section{Existence of positive solution}

In this section we will discuss the existence of positive solution for equation 1.1 with condition 1.2 . Befor starting in prove our result, we interduce the following conditions:

$\left(H_{1}\right) f:(0,1] \times[0, \infty) \longrightarrow[0, \infty)$ is continuous such that $f(\cdot, y(\cdot)) \in C_{1-\gamma}[0,1]$ for any $y \in C_{1-\gamma}[0,1]$.

$\left(H_{2}\right)$ There exist a positive constant $L_{f}$ such that

$$
|f(t, x)-f(t, y)| \leq L_{f}\|x-y\|_{C_{1-\gamma}} .
$$

The following lemmas are fundamental to our results.

Lemma 3.1. If $Q(t):=\int_{\tau}^{1}(s-\tau)^{\alpha-1} d s$, for $\tau \in[0,1]$, then

$$
\frac{Q(\tau)}{\Gamma(\alpha)}<e
$$

Proof. According to the definition of gamma function with some simple computation, we obtain

$$
\begin{aligned}
\frac{Q(\tau)}{\Gamma(\alpha)} & =\frac{\int_{\tau}^{1}(s-\tau)^{\alpha-1} d s}{\int_{0}^{\infty} s^{\alpha-1} e^{-s} d s} \\
& =\frac{\int_{0}^{1-\tau} s^{\alpha-1} d s}{\int_{0}^{\infty} s^{\alpha-1} e^{-s} d s} \leq \frac{e \int_{0}^{1-\tau} s^{\alpha-1} e^{-s} d s}{\int_{0}^{\infty} s^{\alpha-1} e^{-s} d s}<e
\end{aligned}
$$

Lemma 3.2. Assume that $Q(\tau):=\int_{\tau}^{1}(s-\tau)^{\alpha-1} d s$ for $\tau \in[0,1], \mu:=1-\frac{\lambda}{\Gamma(\gamma+1)} \neq 0$, $f \in C_{1-\gamma}[0,1]$ and $y \in C_{1-\gamma}^{\gamma}[0,1]$ exist. A function $y$ is the solution of

$$
\begin{aligned}
D_{0^{+}}^{\alpha, \beta} y(t) & =f(t, y(t)), 0<t \leq 1, \\
I_{0^{+}}^{1-\gamma} y(0) & =\lambda \int_{0}^{1} y(s) d s+d,
\end{aligned}
$$

if and only if $y$ satisfies the fractional integral equation

$$
y(t)=\Lambda t^{\gamma-1}+\frac{\lambda t^{\gamma-1}}{\Gamma(\gamma) \mu} \int_{0}^{1} \frac{Q(\tau)}{\Gamma(\alpha)} f(\tau, y(\tau)) d \tau+\frac{1}{\Gamma(\alpha)} \int_{0}^{t}(t-s)^{\alpha-1} f(s, y(s)) d s,
$$

where $\Lambda:=\left(\frac{\lambda}{\mu \Gamma(\gamma) \Gamma(\gamma+1)}+\frac{1}{\Gamma(\gamma)}\right) d$.

Proof. First, Assume that $y$ satisfies equation (3.2), then by applying $I_{0^{+}}^{\alpha}$ on both side of equation (3.2) and use Lemma 2.9, integral condition, we obtain

$$
y(t)=\frac{\lambda t^{\gamma-1}}{\Gamma(\gamma)} \int_{0}^{1} y(s) d s+\frac{d}{\Gamma(\gamma)} t^{\gamma-1}+\frac{1}{\Gamma(\alpha)} \int_{0}^{t}(t-s)^{\alpha-1} f(s, y(s)) d s .
$$

Set $A:=\int_{0}^{1} y(s) d s$. This the assumption with the equation (3.5) implies

$$
A=\frac{d}{\mu \Gamma(\gamma+1)}+\frac{1}{\mu} \int_{0}^{1} \frac{Q(\tau)}{\Gamma(\alpha)} f(\tau, y(\tau)) d \tau
$$


714 Mohammed A. Almalahi, Satish K. Panchal and Mohammed S. Abdo

substituting (3.6) into (3.5), we attain

$$
y(t)=\Lambda t^{\gamma-1}+\frac{\lambda t^{\gamma-1}}{\Gamma(\gamma) \mu} \int_{0}^{1} \frac{Q(\tau)}{\Gamma(\alpha)} f(\tau, y(\tau)) d \tau+\frac{1}{\Gamma(\alpha)} \int_{0}^{t}(t-s)^{\alpha-1} f(s, y(s)) d s,
$$

for all $t \in(0,1]$.

Conversely, assume that $y$ satisfies (3.4). Applying $I_{0^{+}}^{1-\gamma}$ to both sides of (3.4) yields

$$
\begin{aligned}
I_{0^{+}}^{1-\gamma} y(t)= & \Lambda \Gamma(\gamma)+\frac{\lambda}{\mu} \int_{0}^{1} \frac{Q(\tau)}{\Gamma(\alpha)} f(\tau, y(\tau)) d \tau \\
& +\frac{1}{\Gamma(1-\gamma+\alpha)} \int_{0}^{t}(t-s)^{\alpha-\gamma} f(s, y(s)) d s .
\end{aligned}
$$

Taking the limit at $t \longrightarrow 0^{+}$of last equality and using Lemma 2.10 with $1-\gamma<$ $1-\gamma+\alpha$, we get

$$
I_{0^{+}}^{1-\gamma} y(0)=\Lambda \Gamma(\gamma)+\frac{\lambda}{\mu} \int_{0}^{1} \frac{Q(\tau)}{\Gamma(\alpha)} f(\tau, y(\tau)) d \tau .
$$

From the equation (3.6) with help of the definition of $\Lambda$, it follows that the integral boundary conditions given in (3.3) is satisfied, i.e. $I_{0^{+}}^{1-\gamma} y(0)=\lambda \int_{0}^{1} y(s) d s+d$. Next, applying $D_{0^{+}}^{\gamma}$ to both sides of (3.4) and using lemmas 2.5, 2.8, yields

$$
D_{0^{+}}^{\gamma} y(t)=D_{0^{+}}^{\beta(1-\alpha)} f(t, y(t))
$$

since $y \in C_{1-\gamma}^{\gamma}[0,1]$, by $(2.2)$, we have $D_{0^{+}}^{\gamma} y(t) \in C_{1-\gamma}[0,1]$, therefore

$$
D_{0^{+}}^{\beta(1-\alpha)} f=D I_{0^{+}}^{1-\beta(1-\alpha)} f \in C_{1-\gamma}[0,1] .
$$

For $f \in C_{1-\gamma}[0,1]$, it is clear that $I_{0^{+}}^{1-\beta(1-\alpha)} f \in C_{1-\gamma}^{1}[0,1]$. Consequently, $f$ and $I_{0^{+}}^{1-\beta(1-\alpha)} f$ satisfy Lemma 2.9 .

Now, we apply $I_{0^{+}}^{\beta(1-\alpha)}$ to both side of equation (??), then Lemma 2.9 and definition of Hilfer operator imply that

$$
D_{0^{+}}^{\alpha, \beta} y(t)=f(t, y(t))-\frac{I_{0^{+}}^{1-\beta(1-\alpha)} f(0, y(0))}{\Gamma(\beta(1-\alpha))} t^{\beta(1-\alpha)-1}
$$

By virtue of Lemma 2.10, one can obtain

$$
D_{0^{+}}^{\alpha, \beta} y(t)=f(t, y(t)) .
$$

This completes the proof.

Lemma 3.3. Assume that $\left(H_{1}\right)$ and (3.1) are satisfied. Then the operator $\Delta: \Omega \longrightarrow \Omega$ defined by

$$
\Delta y(t)=\Lambda t^{\gamma-1}+\frac{\lambda t^{\gamma-1}}{\Gamma(\gamma) \mu} \int_{0}^{1} \frac{Q(\tau)}{\Gamma(\alpha)} f(\tau, y(\tau)) d \tau+\frac{1}{\Gamma(\alpha)} \int_{0}^{t}(t-s)^{\alpha-1} f(s, y(s)) d s
$$

is compact. 
Proof. We know that the operator $\Delta: \Omega \longrightarrow \Omega$ is continuous, from fact that $f(t, y(t))$ is continuous and nonnegative. Define bounded set $B_{r} \subset \Omega$ as follows

$$
B_{r}=\left\{y \in \Omega:\|y\|_{C_{1-\gamma}} \leq r\right\} .
$$

The function $f:(0,1] \times B_{r} \longrightarrow \mathbb{R}^{+}$is bounded, then there exist $\xi>0$ such that

$$
0<f(t, y(t)) \leq \xi
$$

In view of equation (3.8), Lemma 3.1, and for all $y \in B_{r}, t \in(0,1]$, we have

$$
\begin{aligned}
& \left|\Delta y(t) t^{1-\gamma}\right| \\
\leq & \Lambda+\frac{\lambda}{\Gamma(\gamma) \mu} \int_{0}^{1} \frac{Q(\tau)}{\Gamma(\alpha)}|f(\tau, y(\tau))| d \tau+\frac{t^{1-\gamma}}{\Gamma(\alpha)} \int_{0}^{t}(t-s)^{\alpha-1}|f(s, y(s))| d s \\
\leq & \Lambda+\frac{\lambda e}{\Gamma(\gamma) \mu} \int_{0}^{1}|f(\tau, y(\tau))| d \tau+\frac{t^{1-\gamma}}{\Gamma(\alpha)} \int_{0}^{t}(t-s)^{\alpha-1}|f(s, y(s))| d s \\
\leq & \Lambda+\frac{\lambda e \xi}{\Gamma(\gamma) \mu}+\frac{t^{1-\gamma+\alpha} \xi}{\Gamma(\alpha+1)},
\end{aligned}
$$

which implies

$$
\|\Delta y\|_{C_{1-\gamma}} \leq\left[\Lambda+\frac{\lambda e \xi}{\Gamma(\gamma) \mu}+\frac{\xi}{\Gamma(\alpha+1)}\right] .
$$

Thus, $\Delta\left(B_{r}\right)$ is uniformly bounded.

Next, we prove that $\Delta\left(B_{r}\right)$ is equicontinuous. Let $y \in B_{r}$. Then for any $\delta, \eta \in$ $(0,1]$ with $0<\delta<\eta \leq 1$, we have

$$
\begin{aligned}
& \left|\frac{\eta^{1-\gamma} \Delta y(\eta)-\delta^{1-\gamma} \Delta y(\delta) \mid}{\Gamma(\alpha)} \int_{0}^{\eta}(\eta-s)^{\alpha-1} f(s, y(s)) d s-\frac{\delta^{1-\gamma}}{\Gamma(\alpha)} \int_{0}^{\delta}(\delta-s)^{\alpha-1} f(s, y(s)) d s\right| \\
\leq & \frac{\eta^{1-\gamma}-\delta^{1-\gamma}}{\Gamma(\alpha)} \int_{0}^{\delta}\left|(\eta-s)^{\alpha-1}-(\delta-s)^{\alpha-1}\right||f(s, y(s))| d s \\
& +\frac{\eta^{1-\gamma}}{\Gamma(\alpha)} \int_{\delta}^{\eta}(\eta-s)^{\alpha-1}|f(s, y(s))| d s \\
\leq & \frac{\left[\eta^{1-\gamma}-\delta^{1-\gamma}\right] \xi}{\Gamma(\alpha)} \int_{0}^{\delta}\left((\delta-s)^{\alpha-1}-(\eta-s)^{\alpha-1}\right) d s \\
& +\frac{\eta^{1-\gamma} \xi}{\Gamma(\alpha)} \int_{\delta}^{\eta}(\eta-s)^{\alpha-1} d s \\
\leq & \frac{\left[\eta^{1-\gamma}-\delta^{1-\gamma}\right] \xi}{\Gamma(\alpha+1)}\left[\left(\delta^{\alpha}-\eta^{\alpha}\right)+(\eta-\delta)^{\alpha}\right]+\frac{\eta^{1-\gamma} \xi}{\Gamma(\alpha+1)}(\eta-\delta)^{\alpha} .
\end{aligned}
$$

By the classical Mean value Theorem, we have

$$
\begin{aligned}
\delta^{\alpha}-\eta^{\alpha} & =\alpha(\delta-\eta) T \\
& \leq \alpha(\delta-\eta)
\end{aligned}
$$


The last inequality with(3.9) implies

$$
\begin{aligned}
& \left|\eta^{1-\gamma} \Delta y(\eta)-\delta^{1-\gamma} \Delta y(\delta)\right| \\
\leq & \frac{\left[\eta^{1-\gamma}-\delta^{1-\gamma}\right] \xi}{\Gamma(\alpha+1)}\left[\alpha(\delta-\eta)+(\eta-\delta)^{\alpha}\right]+\frac{\eta^{1-\gamma} \xi}{\Gamma(\alpha+1)}(\eta-\delta)^{\alpha} \\
\leq & \frac{\left[\eta^{1-\gamma}-\delta^{1-\gamma}\right] \xi}{\Gamma(\alpha+1)}(\eta-\delta)^{\alpha}+\frac{\eta^{1-\gamma} \xi}{\Gamma(\alpha+1)}(\eta-\delta)^{\alpha} \\
\leq & \frac{2 \eta^{1-\gamma} \xi}{\Gamma(\alpha+1)}(\eta-\delta)^{\alpha}-\frac{\delta^{1-\gamma} \xi}{\Gamma(\alpha+1)}(\eta-\delta)^{\alpha} .
\end{aligned}
$$

As $\delta \longrightarrow \eta$ the right-hand side of the preceding inequality is independent of $y$ and tends to zero. So,

$$
\left|\eta^{1-\gamma} \Delta y(\eta)-\delta^{1-\gamma} \Delta y(\delta)\right| \longrightarrow 0, \forall|\eta-\delta| \longrightarrow 0
$$

Hence, $\Delta\left(B_{r}\right)$ is an equicontinuous set. By the Arzela-Ascoli theorem we get that $\Delta\left(B_{r}\right)$ is relatively compact set, which prove that $\Delta: \Omega \longrightarrow \Omega$ is a compact operator.

Definition 3.4. For any $y \in[a, b] \subset \mathbb{R}^{+}$, we define the upper-control function by

$$
G(t, x)=\sup _{a \leq y \leq x} f(t, y)
$$

and the lower-control function by

$$
g(t, x)=\inf _{x \leq y \leq b} f(t, y) .
$$

It is obvious that these functions are nondecreasing on $[a, b]$, i.e.

$$
g(t, x) \leq f(t, y) \leq G(t, x) .
$$

Definition 3.5. Let $\bar{y}, \underline{y} \in \Omega$ such that $0<\underline{y} \leq \bar{y} \leq 1$ satisfy the following Hilfer problem

$$
\begin{aligned}
& D_{0^{+}}^{\alpha, \beta} \bar{y}(t) \geq G(t, x), 0<t \leq 1 \\
& I_{0^{+}}^{1-\gamma} \bar{y}(0) \geq \lambda \int_{0}^{1} \bar{y}(s) d s+d
\end{aligned}
$$

or

$$
\bar{y}(t) \geq \Lambda t^{\gamma-1}+\frac{\lambda t^{\gamma-1}}{\Gamma(\gamma) \mu} \int_{0}^{1} \frac{Q(\tau)}{\Gamma(\alpha)} G(\tau, \bar{y}(\tau)) d \tau+\frac{1}{\Gamma(\alpha)} \int_{0}^{t}(t-s)^{\alpha-1} G(s, \bar{y}(s)) d s,
$$

and

or

$$
\begin{aligned}
& D_{0^{+}}^{\alpha, \beta} \underline{y}(t) \leq g(t, x), 0<t \leq 1 \\
& I_{0^{+}}^{1-\gamma} \underline{y}(0) \leq \lambda \int_{0}^{1} \underline{y}(s) d s+d
\end{aligned}
$$

$$
\underline{y}(t) \leq \Lambda t^{\gamma-1}+\frac{\lambda t^{\gamma-1}}{\Gamma(\gamma) \mu} \int_{0}^{1} \frac{Q(\tau)}{\Gamma(\alpha)} g(\tau, \underline{y}(\tau)) d \tau+\frac{1}{\Gamma(\alpha)} \int_{0}^{t}(t-s)^{\alpha-1} g(s, \underline{y}(s)) d s .
$$


Then the functions $\bar{y}(t)$ and $y(t)$ are called the upper and lower solutions of the Hilfer problem (1.1)-(1.2).

Theorem 3.6. Assume that $\left(H_{1}\right)$ and (3.1) hold. Then there exists at least one positive solution $y(t) \in C_{1-\gamma}[0,1]$ of the Hilfer problem (1.1),(1.2), such that

$$
\underline{y}(t) \leq y(t) \leq \bar{y}(t), \quad 0<t \leq 1 .
$$

where $\bar{y}(t)$ and $\underline{y}(t)$ are upper and lower solutions of Hilfer problem (1.1),(1.2) respectively.

Proof. In view of Lemma (3.2), the solution of problem (1.1)-(1.2)is given by

$$
y(t)=\Lambda t^{\gamma-1}+\frac{\lambda t^{\gamma-1}}{\Gamma(\gamma) \mu} \int_{0}^{1} \frac{Q(\tau)}{\Gamma(\alpha)} f(\tau, y(\tau)) d \tau+\frac{1}{\Gamma(\alpha)} \int_{0}^{t}(t-s)^{\alpha-1} f(s, y(s)) d s
$$

Define

$$
\Upsilon=\{x(t): x(t) \in \Omega, \underline{y}(t) \leq x(t) \leq \bar{y}(t), 0<t \leq 1\}
$$

endowed with the norm $\|x\|=\max _{t \in(0,1]}|x(t)|$, then we have $\|x\| \leq b$. Hence, $\Upsilon$ is a convex, bounded, and closed subset of the Banach space $C_{1-\gamma}[0,1]$. Now, to apply the Schauder fixed point theorem, we divide the proof into several steps as follows:

Step 1. We need to prove that $\Delta: \Omega \longrightarrow \Omega$ is compact .

According to Lemma 3.3, the operator $\Delta: \Omega \longrightarrow \Omega$ is compact. Since $\Upsilon \subset \Omega$, the operator $\Delta: \Upsilon \longrightarrow \Upsilon$ is compact too.

Step 2. We need to prove that $\Delta: \Upsilon \longrightarrow \Upsilon$. Indeed, by the definitions $3.4,3.5$, then for any $x(t) \in \Upsilon$, we have

$$
\begin{aligned}
\Delta x(t) & =\Lambda t^{\gamma-1}+\frac{\lambda t^{\gamma-1}}{\Gamma(\gamma) \mu} \int_{0}^{1} \frac{Q(\tau)}{\Gamma(\alpha)} f(\tau, x(\tau)) d \tau+\frac{1}{\Gamma(\alpha)} \int_{0}^{t}(t-s)^{\alpha-1} f(s, x(s)) d s \\
& \leq \Lambda t^{\gamma-1}+\frac{\lambda t^{\gamma-1}}{\Gamma(\gamma) \mu} \int_{0}^{1} \frac{Q(\tau)}{\Gamma(\alpha)} G(\tau, x(\tau)) d \tau+\frac{1}{\Gamma(\alpha)} \int_{0}^{t}(t-s)^{\alpha-1} G(s, x(s)) d s \\
& \leq \Lambda t^{\gamma-1}+\frac{\lambda t^{\gamma-1}}{\Gamma(\gamma) \mu} \int_{0}^{1} \frac{Q(\tau)}{\Gamma(\alpha)} G(\tau, \bar{y}(\tau)) d \tau+\frac{1}{\Gamma(\alpha)} \int_{0}^{t}(t-s)^{\alpha-1} G(s, \bar{y}(s)) d s \\
& \leq \bar{y}(t) .
\end{aligned}
$$

Also

$$
\begin{aligned}
\Delta x(t) & =\Lambda t^{\gamma-1}+\frac{\lambda t^{\gamma-1}}{\Gamma(\gamma) \mu} \int_{0}^{1} \frac{Q(\tau)}{\Gamma(\alpha)} f(\tau, y(\tau)) d \tau+\frac{1}{\Gamma(\alpha)} \int_{0}^{t}(t-s)^{\alpha-1} f(s, y(s)) d s \\
& \geq \Lambda t^{\gamma-1}+\frac{\lambda t^{\gamma-1}}{\Gamma(\gamma) \mu} \int_{0}^{1} \frac{Q(\tau)}{\Gamma(\alpha)} g(\tau, x(\tau)) d \tau+\frac{1}{\Gamma(\alpha)} \int_{0}^{t}(t-s)^{\alpha-1} g(s, x(s)) d s \\
& \geq \Lambda t^{\gamma-1}+\frac{\lambda t^{\gamma-1}}{\Gamma(\gamma) \mu} \int_{0}^{1} \frac{Q(\tau)}{\Gamma(\alpha)} g(\tau, \underline{y}(\tau)) d \tau+\frac{1}{\Gamma(\alpha)} \int_{0}^{t}(t-s)^{\alpha-1} g(s, \underline{y}(s)) d s \\
& \geq \underline{y}(t) .
\end{aligned}
$$

From (3.11) and (3.12), we conclude that $y(t) \leq \Delta x(t) \leq \bar{y}(t)$, and hence $\Delta x(t) \in \Upsilon$, for $0<t \leq 1$ i. e. $\Delta: \Upsilon \longrightarrow \Upsilon$. 
718 Mohammed A. Almalahi, Satish K. Panchal and Mohammed S. Abdo

In view of the above steps and Schauder fixed point theorem, the problem (1.1)-(1.2) has at least one positive solution $y(t) \in \Upsilon$.

Corollary 3.7. Assume that $f:(0,1] \times[0, \infty) \longrightarrow[0, \infty)$ is continuous, and there exist $A_{1}, A_{2}>0$ such that

$$
A_{1} \leq f(t, y) \leq A_{2}, \quad(t, y) \in(0,1] \times \mathbb{R}^{+} .
$$

Then the Hilfer problem (1.1)-(1.2) has at least one positive solution $y(t) \in \Upsilon$.

Moreover,

$$
\frac{d}{\Gamma(\gamma)} t^{\gamma-1}+\frac{A_{1}}{\Gamma(\alpha+1)} t^{\alpha} \leq y(t) \leq \frac{d}{\Gamma(\gamma)} t^{\gamma-1}+\frac{A_{2}}{\Gamma(\alpha+1)} t^{\alpha}
$$

Proof. From the Definition 3.4 and equation (3.13), we have

$$
A_{1} \leq g(t, y) \leq G(t, y) \leq A_{2} .
$$

Now, we consider the following Hilfer problem

$$
D_{0^{+}}^{\alpha, \beta} \bar{y}(t)=A_{2}, \quad I_{0^{+}}^{1-\gamma} \bar{y}(0)=d .
$$

Then, the Hilfer problem (3.16) has a positive solution

$$
\begin{aligned}
\bar{y}(t) & =\frac{t^{\gamma-1}}{\Gamma(\gamma)} I_{0^{+}}^{1-\gamma} \bar{y}(0)+I_{0^{+}}^{\alpha} A_{2} \\
& =\frac{d}{\Gamma(\gamma)} t^{\gamma-1}+\frac{A_{2}}{\Gamma(\alpha)} \int_{0}^{t}(t-s)^{\alpha-1} d s \\
& =\frac{d}{\Gamma(\gamma)} t^{\gamma-1}+\frac{A_{2}}{\Gamma(\alpha+1)} t^{\alpha} .
\end{aligned}
$$

By (3.15) we conclude that

$\bar{y}(t)=\frac{d}{\Gamma(\gamma)} t^{\gamma-1}+\frac{A_{2}}{\Gamma(\alpha)} \int_{0}^{t}(t-s)^{\alpha-1} d s \geq \frac{d}{\Gamma(\gamma)} t^{\gamma-1}+\frac{1}{\Gamma(\alpha)} \int_{0}^{t}(t-s)^{\alpha-1} G(s, \bar{y}) d s$.

Thus, the function $\bar{y}(t)$ is the upper solution of the Hilfer problem (1.1)-(1.2).

In the similar way, if the Hilfer problem of the type

$$
D_{0^{+}}^{\alpha, \beta} \underline{y}(t)=A_{1}, \quad I_{0^{+}}^{1-\gamma} \underline{y}(0)=d .
$$

Obviously, the Hilfer problem (3.17) has also a positive solution

$$
\begin{aligned}
\underline{y}(t) & =\frac{t^{\gamma-1}}{\Gamma(\gamma)} I_{0^{+}}^{1-\gamma} \underline{y}(0)+I_{0^{+}}^{\alpha} A_{1} \\
& =\frac{d}{\Gamma(\gamma)} t^{\gamma-1}+\frac{A_{1}}{\Gamma(\alpha)} \int_{0}^{t}(t-s)^{\alpha-1} d s \\
& =\frac{d}{\Gamma(\gamma)} t^{\gamma-1}+\frac{A_{1}}{\Gamma(\alpha+1)} t^{\alpha} .
\end{aligned}
$$

Similarly, by (3.15) we infer that

$$
\underline{y}(t)=\frac{d}{\Gamma(\gamma)} t^{\gamma-1}+\frac{A_{1}}{\Gamma(\alpha)} \int_{0}^{t}(t-s)^{\alpha-1} d s \leq \frac{d}{\Gamma(\gamma)} t^{\gamma-1}+\frac{1}{\Gamma(\alpha)} \int_{0}^{t}(t-s)^{\alpha-1} g(s, \bar{y}) d s .
$$


Hence, the function $y(t)$ is the lower solution of the Hilfer problem (1.1)-(1.2).

By Theorem (3.6), we deduce that the problem (1.1)-(1.2) has at least one positive solution $y(t) \in \Omega$, which verifies the inequality (3.14).

\section{Uniqueness of positive solution}

In this section, we will demonstrate the uniqueness of the positive solution using the Banach contraction principle.

Theorem 4.1. Assume that $f:(0,1] \times[0, \infty) \longrightarrow[0, \infty)$ is continuous, the condition $\left(H_{2}\right)$ and the inequality (3.1) hold. If

$$
\left(\frac{\lambda e}{\Gamma(\gamma) \mu}+\frac{1}{\Gamma(\alpha+1)}\right) L_{f}<1
$$

Then the problem (1.1)-(1.2) has a unique positive solution in $\Upsilon$.

Proof. According to Theorem (3.6), the problem (1.1)-(1.2) has at least one positive solution in $\Upsilon$ as the form

$$
\begin{aligned}
y(t) \longrightarrow & \Delta y(t)=\Lambda t^{\gamma-1}+\frac{\lambda t^{\gamma-1}}{\Gamma(\gamma) \mu} \int_{0}^{1} \frac{Q(\tau)}{\Gamma(\alpha)} f(\tau, y(\tau)) d \tau \\
& +\frac{1}{\Gamma(\alpha)} \int_{0}^{t}(t-s)^{\alpha-1} f(s, y(s)) d s .
\end{aligned}
$$

Now, we need only to proof that the operator $\Delta$ is contraction mapping on $\Upsilon$. Indeed, for any $y_{1}, y_{2} \in \Upsilon$ and $t \in(0,1]$, we have

$$
\begin{aligned}
& \left|t^{1-\gamma} \Delta y_{1}(t)-t^{1-\gamma} \Delta y_{2}(t)\right| \\
\leq & \frac{\lambda}{\Gamma(\gamma) \mu} \int_{0}^{1} \frac{Q(\tau)}{\Gamma(\alpha)} \mid f\left(\tau, y_{1}(\tau)\right)-f\left(\tau, y_{2}(\tau) \mid d \tau\right. \\
& +\frac{t^{1-\gamma}}{\Gamma(\alpha)} \int_{0}^{t}(t-s)^{\alpha-1}\left|f\left(s, y_{1}(s)\right)-f\left(s, y_{2}(s)\right)\right| d s \\
\leq & \frac{\lambda e}{\Gamma(\gamma) \mu} \int_{0}^{1} \mid f\left(\tau, y_{1}(\tau)\right)-f\left(\tau, y_{2}(\tau) \mid d \tau\right. \\
& +\frac{t^{1-\gamma}}{\Gamma(\alpha)} \int_{0}^{t}(t-s)^{\alpha-1}\left|f\left(s, y_{1}(s)\right)-f\left(s, y_{2}(s)\right)\right| d s \\
\leq & \frac{\lambda e}{\Gamma(\gamma) \mu} \int_{0}^{1} L_{f}\left\|y_{1}-y_{2}\right\|_{C_{1-\gamma}} d \tau+\frac{t^{1-\gamma}}{\Gamma(\alpha)} \int_{0}^{t}(t-s)^{\alpha-1} L_{f}\left\|y_{1}-y_{2}\right\|_{C_{1-\gamma}} d s \\
\leq & \frac{\lambda e L_{f}}{\Gamma(\gamma) \mu}\left\|_{1}-y_{2}\right\|_{C_{1-\gamma}}+\frac{t^{1-\gamma+\alpha}}{\Gamma(\alpha+1)} L_{f}\left\|y_{1}-y_{2}\right\|_{C_{1-\gamma}} \\
\leq & \left(\frac{\lambda e}{\Gamma(\gamma) \mu}+\frac{1}{\Gamma(\alpha+1)}\right) L_{f}\left\|y_{1}-y_{2}\right\|_{C_{1-\gamma}}
\end{aligned}
$$

The hypothesis (4.1) shows that $\Delta$ is a contraction mapping. The conclusion from the Banach contraction principle that the Hilfer problem (1.1)-(1.2) has a unique positive solution $u(t) \in C_{1-\gamma}[0,1]$. 


\section{An example}

In this part, we present an example to illustrate our result.

Example 5.1. Consider the following nonlinear Hilfer fractional problem

$$
\begin{aligned}
D_{0^{+}}^{\frac{1}{2}, \frac{1}{3}} y(t) & =\frac{3}{5}\left(t^{\frac{1}{2}}|\sin y(t)|+1\right), \quad t \in(0,1] \\
I_{0^{+}}^{\frac{2}{3}} y(0) & =1
\end{aligned}
$$

where $\alpha=\frac{1}{2}, \beta=\frac{1}{3}, \gamma=\frac{2}{3}, \lambda=0, d=1$ and $f(t, y(t))=\frac{3}{5}\left(t^{\frac{1}{2}}|\sin y(t)|+1\right)$. It is easy to see that

$$
t^{\frac{1}{3}} f(t, y(t))=\frac{3}{5}\left(t^{\frac{5}{6}}|\sin y(t)|+t^{\frac{1}{3}}\right) \in C[0,1] .
$$

Hence $f(t, y(t)) \in C_{\frac{1}{3}}[0,1]$, which means that $f$ satisfies $\left(\mathrm{H}_{1}\right)$. Next, we show that $f$ satisfies $\left(\mathrm{H}_{2}\right)$. In fact, for any $y_{1}, y_{2} \in C_{\frac{1}{3}}[0,1]$ and $t \in(0,1]$, we have

$$
\begin{aligned}
\left|f\left(t, y_{1}(t)\right)-f\left(t, y_{2}(t)\right)\right| & \leq\left|\frac{3}{5} t^{\frac{1}{2}} \sin y_{1}(t)-\frac{3}{5} t^{\frac{1}{2}} \sin y_{2}(t)\right| \\
& \leq \frac{3}{5} t^{\frac{1}{6}}\left|t^{\frac{1}{3}} \sin y_{1}(t)-t^{\frac{1}{3}} \sin y_{2}(t)\right| \\
& \leq \frac{3}{5}\left\|y_{1}-y_{2}\right\|_{C_{\frac{1}{3}}}=L_{f}\left\|y_{1}-y_{2}\right\|_{C_{\frac{1}{3}}}
\end{aligned}
$$

Since $f$ is continuous and

$$
\frac{3}{5} \leq f(t, y) \leq \frac{6}{5}, \quad(t, y) \in(0,1] \times \mathbb{R}^{+} .
$$

Then the Hilfer problem (5.1) has a positive solution which verifies $\underline{y}(t) \leq y(t) \leq \bar{y}(t)$ where

$$
\bar{y}(t)=\frac{1}{\Gamma\left(\frac{2}{3}\right)} t^{\frac{-1}{3}}+\frac{6}{5 \Gamma\left(\frac{3}{2}\right)} t^{\frac{1}{2}}
$$

and

$$
\underline{y}(t)=\frac{1}{\Gamma\left(\frac{2}{3}\right)} t^{\frac{-1}{3}}+\frac{3}{5 \Gamma\left(\frac{3}{2}\right)} t^{\frac{1}{2}} .
$$

are respectively the upper and lower solutions of Hilfer problem (5.1). Furthermore, by simple computations, the condition (4.1) also is satisfied, i.e.

$$
\left(\frac{\lambda e}{\Gamma(\gamma) \mu}+\frac{1}{\Gamma(\alpha+1)}\right) L_{f}=\frac{1}{\Gamma\left(\frac{3}{2}\right)} \frac{3}{5} \simeq 0.7<1
$$

Thus, since all the hypotheses in Theorems 3.6, 4.1 are fulfilled, our results can be applied to the Hilfer problem. 


\section{Conclusion}

This paper studies the existence and uniqueness of positive solution of the nonlinear fractional differential equation with integral boundary condition and Hilfer fractional derivative operator. The proof of the main results relies on the Schauder fixed point theorem, Banach contraction mapping principle and technique of upper and lower solution.

The method of constructing a pair of upper and lower control functions with respect to the nonlinear term without monotone demand provides a new efficient technique to handle the nonlinear structure. This method is a tremendous tool for solving nonlinear differential equations in applied mathematics. The obtained results extend some known results in the literature. An example is introduced to illustrate the main results of this paper.

\section{References}

[1] Abdo, M.S., Wahash, H.A., Panchal, S.K., Positive solution of a fractional differential equation with integral boundary conditions, Journal of Applied Mathematics and Computational Mechanics, 17(2018), no. 3.

[2] Almalahi, M.A., Abdo, M.S., Panchal, S.K., Existence and Ulam-Hyers-Mittag-Leffler stability results of $\psi$-Hilfer nonlocal Cauchy problem, Rend. Circ. Mat. Palermo, II. Ser (2020).

[3] Ardjouni, A., Positive solutions for nonlinear Hadamard fractional differential equations with integral boundary conditions, AIMS Mathematics, 4(2019), 1101-1113.

[4] Ardjouni, A., Djoudi, A., Existence and uniqueness of positive solutions for first-order nonlinear Liouville-Caputo fractional differential equations, São Paulo Journal of Mathematical Sciences, (2019), 1-10.

[5] Boulares, H., Ardjouni, A., Laskri, Y., Positive solutions for nonlinear fractional differential equations, Positivity, 21(3)(2017), 1201-1212.

[6] Furati, K.M., Kassim, M.D., Existence and uniqueness for a problem involving Hilfer fractional derivative, Comput. Math. Applic., 64(2012), 1616-1626.

[7] Hilfer R., Applications of Fractional Calculus in Physics, World Scientific Publishing Co., Inc., River 27 Edge, NJ, Singapore, 2000.

[8] Kilbas, A.A., Srivastava, H.M., Trujillo, J.J., Theory and Applications of Fractional Differential Equations, North-Holland Mathematics Studies, Elsevier, Amsterdam, 207, 2006.

[9] Long, T., Li, C., He, J., Existence of positive solutions for period BVPs with Hilfer derivative, Journal of Applied Mathematics and Computing, 60(2019), no. 1-2, 223-236.

[10] Miller, K.S., Ross, B., An Introduction to the Fractional Calculus and Differential Equations, New York, John Wiley, 1993.

[11] Nan, L.I., Changyou, W.A.N.G., New existence results of positive solution for a class of nonlinear fractional differential equations, Acta Mathematica Scientia, 33(2013), no. 3, 847-854.

[12] Podlubny, I., Fractional differential equations: an introduction to fractional derivatives, Fractional Differential Equations, to Methods of Their Solution and Some of Their Applications, Elsevier, 198, 1998. 
[13] Wang, F., Liu, L., Kong, D., Wu, Y., Existence and uniqueness of positive solutions for a class of nonlinear fractional differential equations with mixed-type boundary value conditions, Nonlinear Analysis: Modelling and Control, 24(2019), no. 1, 73-94.

[14] Zhang, S., The existence of a positive solution for a nonlinear fractional differential equation, Journal of Mathematical Analysis and Applications, 252(2000), no. 2, 804812.

Mohammed A. Almalahi

Department of Mathematics,

Dr. Babasaheb Ambedkar Marathwada University,

Aurangabad, (M.S.), 431001, India

e-mail: aboosama736242107@gmail.com

Satish K. Panchal

Department of Mathematics,

Dr. Babasaheb Ambedkar Marathwada University,

Aurangabad, (M.S),431001, India

e-mail: drpanchalsk@gmail.com

Mohammed S. Abdo

Department of Mathematics,

Dr. Babasaheb Ambedkar Marathwada University,

Aurangabad, (M.S.), 431001, India

e-mail: msabdo1977@gmail.com 